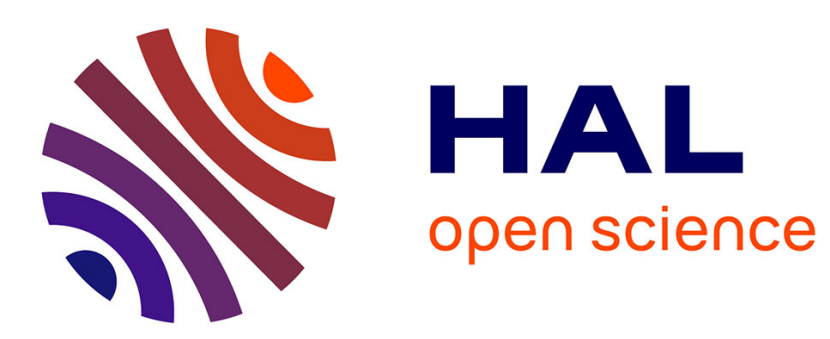

\title{
SHARP CONDITION FOR THE LIOUVILLE PROPERTY IN A CLASS OF NONLINEAR ELLIPTIC INEQUALITIES
}

Philippe Souplet

\section{- To cite this version:}

Philippe Souplet. SHARP CONDITION FOR THE LIOUVILLE PROPERTY IN A CLASS OF NONLINEAR ELLIPTIC INEQUALITIES. 2020. hal-02446681

\section{HAL Id: hal-02446681 \\ https://hal.science/hal-02446681}

Preprint submitted on 21 Jan 2020

HAL is a multi-disciplinary open access archive for the deposit and dissemination of scientific research documents, whether they are published or not. The documents may come from teaching and research institutions in France or abroad, or from public or private research centers.
L'archive ouverte pluridisciplinaire HAL, est destinée au dépôt et à la diffusion de documents scientifiques de niveau recherche, publiés ou non, émanant des établissements d'enseignement et de recherche français ou étrangers, des laboratoires publics ou privés. 


\title{
SHARP CONDITION FOR THE LIOUVILLE PROPERTY IN A CLASS OF NONLINEAR ELLIPTIC INEQUALITIES
}

\author{
Philippe SOUPLET
}

Université Sorbonne Paris Nord,

CNRS UMR 7539, Laboratoire Analyse, Géométrie et Applications

93430 Villetaneuse, France. E-mail: souplet@math.univ-paris13.fr

\begin{abstract}
We study a class of elliptic inequalities which arise in the study of blow-up rate estimates for parabolic problems, and obtain a sharp existence/nonexistence result. Namely, for any $p \geq 1$, we show that the inequality $\Delta u+u^{p} \leq \varepsilon$ in $\mathbb{R}^{n}$ with $u(0)=1$ admits a radial, positive nonincreasing solution for all $\varepsilon>0$, if and only if $n \geq 2$. This solves a problem left open in [Souplet \& Tayachi, Colloq. Math. 2001]. The result stands in contrast with the classical case $\varepsilon=0$.
\end{abstract}

AMS Classification: Primary: 35J60, 35B08, 35B53; Secondary; 35K55, 35B44.

Keywords: elliptic inequalities, Liouville property, parabolic inequalities, blowup rate.

\section{Introduction and main results}

The purpose of this note is to discuss certain elliptic inequalities which arise in the study of blow-up rate estimates for parabolic problems. Blow-up rate estimates for nonlinear parabolic equations are a classical and intensively studied topic. We refer to the monograph [10] and the numerous references therein, especially for the case of the model equation

$$
v_{t}-\Delta v=v^{p} \quad(p>1) .
$$

It is to be noted that upper blow-up estimates are usually much more delicate to obtain than lower estimates. Without entering into details, let us just recall that for positive solutions of the Cauchy problem associated with (1.1), the lower estimate

$$
\inf _{t \in(0, T)}(T-t)^{1 /(p-1)}\|v(t)\|_{\infty}>0
$$

is true whenever the maximal existence time $T$ of $v$ is finite. As for the upper estimate

$$
\sup _{t \in(0, T)}(T-t)^{1 /(p-1)}\|v(t)\|_{\infty}<\infty
$$

it is true whenever $p<p_{S}:=(n+2) /(n-2)_{+}$(see [5]) but it may fail for $p=p_{S}$ or for some larger values of $p$ (see e.g. $[6,9,11,1,2,3]$ ).

In [12], among other things, we asked whether upper blow-up rate estimates can still be established for parabolic inequalities, instead of equations. We showed in particular the following theorem:

Theorem A. (i) Let $p>1, R, T>0$ and set $Q_{T}=(0, T) \times(-R, R)$. Let $0 \leq v \in C^{1,2}\left(Q_{T}\right)$ satisfy

$$
v_{t}-v_{x x} \geq v^{p} \quad \text { in } Q_{T},
$$

where $v$ is symmetric as a function of $r=|x|$ and satisfies $v_{t} \geq 0, v_{r} \leq 0$ in $Q_{T}$. Then $v$ satisfies the estimate

$$
\limsup _{t \rightarrow T}(T-t)^{1 /(p-1)}\|v(t)\|_{\infty}<\infty .
$$

(ii) If we relax the assumption $v_{t} \geq 0$ above, then $v$ still satisfies the weaker estimate

$$
\liminf _{t \rightarrow T}(T-t)^{1 /(p-1)}\|v(t)\|_{\infty}<\infty .
$$


Among the known methods to prove upper blow-up rate estimates for parabolic equations, a particularly powerful approach is to combine rescaling arguments with Liouville-type nonexistence results (see e.g. [13, 5, 7]). The proof of Theorem A in [12] for one-dimensional parabolic inequalities was based on a modification of a rescaling argument from [13], and the key nonexistence result used in the proof was the following differential inequality lemma (cf. [12, Lemma 3.4]):

Lemma B. Let $p \geq 1$. Then, for all sufficiently small $\varepsilon>0$, the problem

$$
\left\{\begin{array}{l}
u_{r r}+u^{p} \leq \varepsilon, \quad r>0 \\
u(0)=1, \quad u_{r}(0)=0
\end{array}\right.
$$

does not admit any positive nonincreasing solution $u \in C^{2}([0, \infty))$.

As noted in [12], the only reason for the limitation $n=1$ in Theorem A came from our inability to establish a higher dimensional version of Lemma B, namely for the elliptic inequality

$$
\Delta u+u^{p} \leq \varepsilon, \quad x \in \mathbb{R}^{n},
$$

and the possibility of such an extension was left there as an open problem (cf. [12, Remark 3.2]). The aim of this note is to show that Lemma B is actually false in any dimension $n \geq 2$. We shall prove the following theorem:

Theorem 1. Let $p \geq 1$ and $n \geq 2$. Then, for each $\varepsilon>0$, the problem

$$
\left\{\begin{array}{l}
\Delta u+u^{p} \leq \varepsilon, \quad x \in \mathbb{R}^{n} \\
u(0)=1
\end{array}\right.
$$

possesses a radial, positive nonincreasing solution $u \in C^{2}\left(\mathbb{R}^{n}\right)$.

As a consequence of Lemma $\mathrm{B}$, we thus see that Theorem 1 holds if and only if $n \geq 2$. In fact, keeping in mind the form of the $n$-dimensional radial Laplacian $\partial_{r}^{2}+(n-1) r^{-1} \partial_{r}$, we can show that Theorem 1 is sharp even with respect to "fractional" dimensions, by extending Lemma B as follows.

Theorem 2. Let $p \geq 1$ and $\alpha \in(0,1)$. Then, for all sufficiently small $\varepsilon>0$, the problem

$$
\left\{\begin{array}{l}
u_{r r}+\alpha \frac{u_{r}}{r}+u^{p} \leq \varepsilon, \quad r>0, \\
u(0)=1, \quad u_{r}(0)=0,
\end{array}\right.
$$

does not admit any positive nonincreasing solution $u \in C^{2}([0, \infty))$.

It is also interesting to compare Theorem 1 with well-known results concerning the case $\varepsilon=0$, for which the situation is completely different. Indeed (see e.g. [4, 8]) the elliptic inequality

$$
\Delta u+u^{p} \leq 0, \quad x \in \mathbb{R}^{n}
$$

has no positive solution if $(n-2) p \leq n$, whereas it admits positive, radial nonincreasing classical solutions (for any prescribed value at $x=0$ ) whenever $n \geq 3$ and $p>n /(n-2)$. Accordingly, it can be seen that the solutions $u(x)=u_{\varepsilon}(x)$ constructed in the proof of Theorem 1 vanish for all $x \in \mathbb{R}^{n} \backslash\{0\}$ as $\varepsilon \rightarrow 0$.

Remarks. (i) We note that Theorem 1 includes the linear case $p=1$. This provides a "quasi-eigenfunction" existence result which seems to be of some independent interest.

(ii) Actually the conclusion of Theorem 2 can be made a bit stronger (as was the conclusion of Lemma B in [12]): problem (1.3) on the interval $[0, R]$ does not have any positive nonincreasing solution, whenever $R \geq R_{0}$ and $\varepsilon \leq \varepsilon_{0}$, where $R_{0}$ and $\varepsilon_{0}>0$ depend only on $p, \alpha$.

(iii) We still do not know whether or not Theorem A itself is true for $n \geq 2$. At least Theorem 1 tells us that the rescaling method used in [12] for $n=1$ cannot be applied in dimension $n \geq 2$. 


\section{Proofs}

Let us outline the proof of Theorem 1. First of all, it suffices to establish the result for $n=2$ and $p=1$ (which turns out to be the most difficult case). Indeed, radial solutions $u=$ $u(r)=u(|x|)$ of problem (1.2) solve (1.3) with $\alpha=n-1$ and, since we are interested in solutions such that $u_{r} \leq 0$, hence $0<u \leq 1$, any solution for $n=2$ solves the same problem for $n>2$. And of course, it also suffices to establish the result for all sufficiently small $\varepsilon>0$. We first construct a solution $w$ of

$$
\left\{\begin{array}{l}
w^{\prime \prime}+\frac{w^{\prime}}{r}+w \leq \varepsilon, \quad r>0, \\
w(0)>1, \quad w^{\prime}(0)=0
\end{array}\right.
$$

which is $C^{1}$ and piecewise $C^{2}$. It will be eventually regularized to a $C^{2}$ solution (and normalized).

Formally, the main idea behind the construction of $w$ is to look for a function connecting the central point $\left\{w \sim 1, w^{\prime}=0\right\}$ at $r=0$ to a small constant $\left\{w=0^{+}, w^{\prime}=0\right\}$ at some $r>0$, and satisfying (2.1), and this connection is achieved in three steps as follows:

$$
\left(\begin{array}{c}
w(0) \sim 1 \\
w^{\prime}(0)=0
\end{array}\right) \longrightarrow\left(\begin{array}{c}
w(a) \sim 1 \\
w^{\prime}(a) \ll-1
\end{array}\right) \longrightarrow\left(\begin{array}{c}
w(b) \sim 0^{+} \\
w^{\prime}(b) \sim 0^{-}
\end{array}\right) \longrightarrow\left(\begin{array}{c}
w(d) \sim 0^{+} \\
w^{\prime}(d)=0
\end{array}\right)
$$

with $a \sim 0^{+}, b, d=O(1)$, as $\varepsilon \rightarrow 0$. In the intervals $(0, a)$ and $(b, d)$, we respectively impose

$$
w^{\prime \prime}+\frac{w^{\prime}}{r}=\text { const } \ll-1 \quad \text { and } \quad w^{\prime \prime}+\frac{w^{\prime}}{r}=\text { const }=\frac{\varepsilon}{2}
$$

(cf. (2.5)), leading to explicit simple choices of $w$. In the central interval $(a, b)$, which is more delicate, we need a slightly superharmonic function with $w^{\prime} \ll-1$ near $r=a$. Recalling that the fundamental solution of the two dimensional Laplacian is in $-\log r$, it turns out that a good choice for the main term is in $\log |\log r|$. The continuity of $w, w^{\prime}$ at $r=a, b, d$ and the inequality (2.1) in each interval can then be verified by suitable (and rather involved) choice of the parameters entering in the expression of $w$. The piecewise shape of $w$ is depicted in Fig. 1, and we will find the following values for the corresponding parameters in terms of $\varepsilon \sim 0^{+}$:

$$
a=\exp \left[-\exp \left(\varepsilon^{-1}|\log \varepsilon|\right)\right], \quad b=\frac{1}{e}, \quad d:=\sqrt{\frac{1}{e^{2}}+\frac{4}{|\log \varepsilon|}}, \quad \theta \sim \frac{2 e^{2} \varepsilon}{|\log \varepsilon|^{2}} .
$$

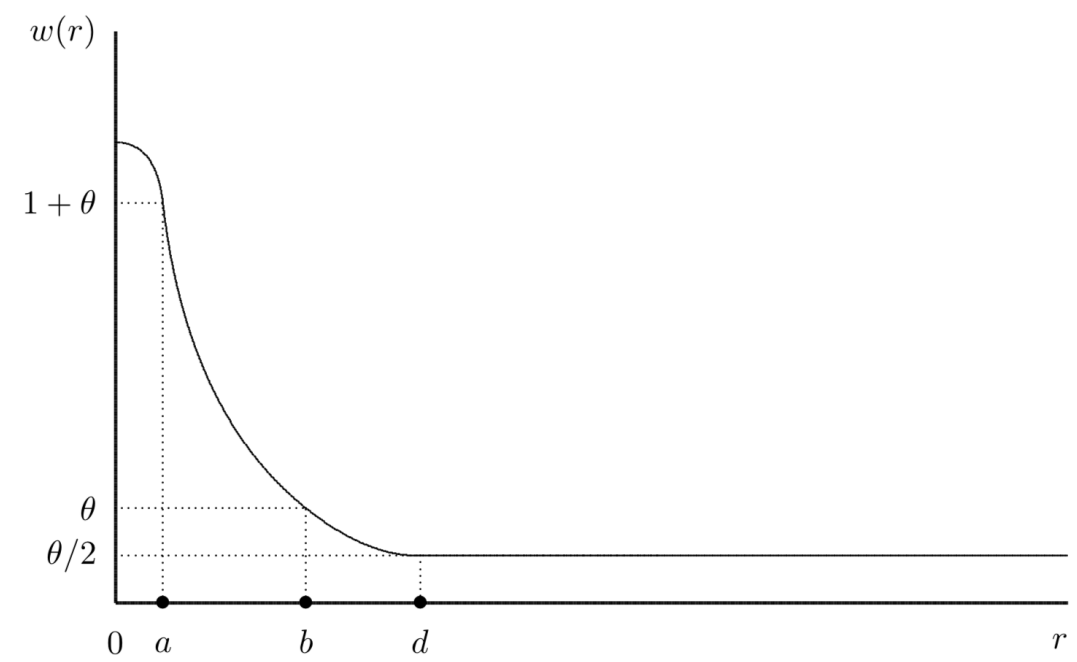

Figure 1. The shape of the function $w(r)$. 
Proof of Theorem 1. Step 1. Definition of $w$. As already noted, we may assume $n=2$ and $p=1$, and it suffices to establish the result for all sufficiently small $\varepsilon>0$. We introduce three parameters $a, d, \theta>0$ which will be subsequently chosen such that

$$
0<a<1 / e<d
$$

and we set

$$
k=\frac{1}{\log |\log a|} .
$$

We then define a function $w$ as follows

$$
w(r)= \begin{cases}1+\theta+\frac{k}{2|\log a|}\left(1-\frac{r^{2}}{a^{2}}\right), & 0 \leq r \leq a \\ 1+\theta+k(\log |\log r|-\log |\log a|)=\theta+\frac{\log |\log r|}{\log |\log a|}, & a<r \leq 1 / e \\ \theta-\left(k+\frac{\varepsilon}{4 e^{2}}\right) \log (e r)+\frac{\varepsilon}{8}\left(r^{2}-\frac{1}{e^{2}}\right), & 1 / e<r \leq d \\ \frac{\theta}{2}, & r>d .\end{cases}
$$

Step 2. $C^{1}$ regularity, positivity and monotonicity of $w$. We have

$$
w^{\prime}(r)= \begin{cases}-\frac{k r}{a^{2}|\log a|}, & 0 \leq r<a \\ \frac{k}{r \log r}=-\frac{k}{r|\log r|}, & a<r<1 / e \\ -\left(k+\frac{\varepsilon}{4 e^{2}}\right) \frac{1}{r}+\frac{\varepsilon r}{4}, & 1 / e<r<d \\ 0, & r>d .\end{cases}
$$

The continuity of $w$ and $w^{\prime}$ at $r=a$ and $1 / e$ is clear from the definition. To ensure the continuity of $w^{\prime}$ at $r=d$, i.e.

$$
-\left(k+\frac{\varepsilon}{4 e^{2}}\right) \frac{1}{d}+\frac{\varepsilon d}{4}=0 \Longleftrightarrow\left(4 k+\frac{\varepsilon}{e^{2}}\right)=\varepsilon d^{2}
$$

we choose

$$
d:=\sqrt{\frac{1}{e^{2}}+\frac{4 k}{\varepsilon}}>\frac{1}{e} .
$$

As for the continuity of $w$ at $r=d$, it is equivalent to

$$
\theta-\left(k+\frac{\varepsilon}{4 e^{2}}\right) \log (e d)+\frac{\varepsilon}{8}\left(d^{2}-e^{-2}\right)=\frac{\theta}{2} \Longleftrightarrow\left(k+\frac{\varepsilon}{4 e^{2}}\right) \log (e d)-\frac{\varepsilon}{8}\left(d^{2}-e^{-2}\right)=\frac{\theta}{2}
$$

which leads to the choice

$$
\theta:=\left(k+\frac{\varepsilon}{4 e^{2}}\right)\left[2+\log \left(\frac{1}{e^{2}}+\frac{4 k}{\varepsilon}\right)\right]-k .
$$

We need $\theta$ to be positive. For later purposes we actually want to guarantee that

$$
0<\theta \leq \min \left\{\frac{\varepsilon}{2}, \frac{c k}{|\log \varepsilon|}\right\}
$$


for some positive constant $c$, independent of $\varepsilon>0$ small. To this end, we select $a \in(0,1 / e)$ such that

$$
k \equiv \frac{1}{\log |\log a|}=\frac{\varepsilon}{|\log \varepsilon|},
$$

i.e.

$$
a=\exp \left[-\exp \left(\varepsilon^{-1}|\log \varepsilon|\right)\right]
$$

Since

$$
\theta=\left(k+\frac{\varepsilon}{4 e^{2}}\right)\left[2+\log \left[\frac{1}{e^{2}}\left(1+\frac{4 k e^{2}}{\varepsilon}\right)\right]-k=\left(k+\frac{\varepsilon}{4 e^{2}}\right) \log \left[1+\frac{4 k e^{2}}{\varepsilon}\right]-k,\right.
$$

using (2.3), we have

$$
\begin{aligned}
\theta & =\left(k+\frac{\varepsilon}{4 e^{2}}\right)\left[\frac{4 k e^{2}}{\varepsilon}-\frac{1}{2}\left(\frac{4 k e^{2}}{\varepsilon}\right)^{2}+O\left(\frac{k^{3}}{\varepsilon^{3}}\right)\right]-k \\
& =\frac{4 k^{2} e^{2}}{\varepsilon}-\frac{1}{2}\left(\frac{4 k e^{2}}{\varepsilon}\right)^{2} \frac{\varepsilon}{4 e^{2}}-\frac{1}{2}\left(\frac{4 k e^{2}}{\varepsilon}\right)^{2} k+O\left(\frac{k^{3}}{\varepsilon^{2}}\right) \\
& =\frac{2 k^{2} e^{2}}{\varepsilon}+O\left(\frac{k^{3}}{\varepsilon^{2}}\right) \sim \frac{2 e^{2} \varepsilon}{|\log \varepsilon|^{2}},
\end{aligned}
$$

as $\varepsilon \rightarrow 0^{+}$, hence $(2.2)$.

The above in particular shows that $w$ is a $C^{1}$ positive nonincreasing function on $[0, \infty)$. Also, since $w^{\prime}(0)=0$, the function $w(|x|)$ belongs to $C^{1}\left(\mathbb{R}^{2}\right)$.

Step 3. Elliptic inequality for $w$. We shall prove that

$$
\Delta_{\text {rad }} w+w:=w^{\prime \prime}+\frac{w^{\prime}}{r}+w \leq \varepsilon, \quad \text { for all } r \in(0, \infty) \backslash\{a, 1 / e, d\} .
$$

We compute

$$
\Delta_{r a d} w= \begin{cases}-\frac{2 k}{a^{2}|\log a|}, & 0<r<a \\ -\frac{k}{r^{2}(\log r)^{2}}, & a<r<1 / e \\ \frac{\varepsilon}{2}, & 1 / e<r<d \\ 0, & r>d,\end{cases}
$$

hence

$$
\Delta_{\text {rad }} w+w \leq \begin{cases}-\frac{2 k}{a^{2}|\log a|}+\left[1+\theta+\frac{k}{2|\log a|}\right]=: \eta_{a}, & 0<r<a \\ -\frac{k}{r^{2}(\log r)^{2}}+[\theta+k \log |\log r|], & a<r<1 / e \\ \frac{\varepsilon}{2}+\theta \leq \varepsilon, & 1 / e<r<d \\ \frac{\theta}{2}<\varepsilon, & r>d,\end{cases}
$$

where we used (2.2). By (2.3), we have

$$
\eta_{a} \leq-\frac{2}{a^{2}|\log a| \log |\log a|}+\left[2+\frac{1}{2|\log a| \log |\log a|}\right]<0
$$


for $\varepsilon>0$ sufficiently small.

It remains to verify $(2.4)$ in the interval $(a, 1 / e)$. Owing to $(2.2)$, we have

$$
A(r):=-\frac{k}{r^{2}(\log r)^{2}}+[\theta+k \log |\log r|] \leq k\left[\log |\log r|-\frac{1}{r^{2}(\log r)^{2}}+\frac{c}{|\log \varepsilon|}\right] .
$$

We claim that $A(r) \leq 0$ in the interval $(a, 1 / e)$ for $\varepsilon>0$ small. To this end it suffices to show the elementary inequality

$$
\sup _{r \in(0,1 / e]}\left[\log |\log r|-\frac{1}{r^{2}(\log r)^{2}}\right]<0 .
$$

By the change of variable $r=1 / s$, this is equivalent to

$$
\sup _{s \geq e} g(s)<0, \quad \text { where } g(s):=\log (\log s)-\frac{s^{2}}{(\log s)^{2}} .
$$

To show $(2.7)$, set $h(s):=\log s-s^{\frac{2}{3}}$. We have $h^{\prime}(s)=s^{-1}-\frac{2}{3} s^{-\frac{1}{3}}=s^{-1}\left[1-\frac{2}{3} s^{\frac{2}{3}}\right]<0$ on $[e, \infty)$, due to $\frac{2}{3} e^{\frac{2}{3}}>\frac{2}{3}\left(1+\frac{2}{3}\right)>1$. Since $h(e)<0$, it follows that

$$
1 \leq \log s<s^{\frac{2}{3}}, \quad s \geq e .
$$

Consequently,

$$
0 \leq \log (\log s)<\log \left(s^{\frac{2}{3}}\right)=\frac{2}{3} \log s<\frac{2}{3} s^{\frac{2}{3}}, \quad s \geq e,
$$

so that

$$
(\log s)^{2} \log (\log s)<\frac{2}{3} s^{\frac{2}{3}} s^{\frac{4}{3}}<s^{2}, \quad s \geq e,
$$

hence $g(s)<0$ for all $s \geq e$. Since $\lim _{s \rightarrow \infty} g(s)=-\infty$, property (2.7), and hence (2.6), follows.

The proof of (2.4) is complete.

Step 4. Regularisation and normalisation. Let $0 \leq \rho \in C_{0}^{\infty}\left(\mathbb{R}^{2}\right)$ be such that $\operatorname{Supp}(\rho) \subset B_{a}$ and $\int_{\mathbb{R}^{2}} \rho d x=1$, with $\rho$ radially symmetric nonincreasing. Since $W(x):=$ $w(|x|)$ is $C^{1}$ and piecewise $C^{2}$ (outside of the circles $|x|=a,|x|=1 / e$ and $|x|=d$ ), it follows from the standard jumps formula for distribution derivatives that $W \in H_{l o c}^{2}\left(\mathbb{R}^{2}\right)$. Setting $V=W * \rho$, we thus have $V \in C^{\infty}\left(\mathbb{R}^{2}\right)$ and

$$
\Delta V=W * \Delta \rho=\rho * \Delta W,
$$

hence, by (2.4),

$$
\Delta V+V=(\Delta W+W) * \rho \leq \varepsilon * \rho=\varepsilon .
$$

Moreover, $V$ is radially symmetric nonincreasing and, since $W(y) \geq 1$ for $|y| \leq a$, we have

$$
\alpha:=V(0)=\int_{\mathbb{R}^{2}} W(-y) \rho(y) d y=\int_{|y| \leq a} W(-y) \rho(y) d y \geq \int_{|y| \leq a} \rho(y) d y=1 .
$$

Finally setting $u=\alpha^{-1} V$, we have $\Delta u+u=\alpha^{-1}(\Delta V+V) \leq \alpha^{-1} \varepsilon \leq \varepsilon$ in $\mathbb{R}^{2}$ and $u$ has all the required properties. The proof of Theorem 1 is complete.

Proof of Theorem 2. We adapt the method of proof of Lemma B in [12] (which treated the case $\alpha=0)$. Assume for contradiction that such a solution $u$ exists. Writing (1.3) as $\left(r^{\alpha} u_{r}\right)_{r}+r^{\alpha} u^{p} \leq r^{\alpha} \varepsilon$, we have

$$
r^{\alpha} u_{r}(r)+\int_{s}^{r} \tau^{\alpha} u^{p}(\tau) d \tau \leq s^{\alpha} u_{r}(s)+\varepsilon \frac{r^{\alpha+1}-s^{\alpha+1}}{\alpha+1}, \quad 0 \leq s<r<\infty,
$$


hence

$$
u_{r}(r)+\int_{s}^{r}\left(\frac{\tau}{r}\right)^{\alpha} u^{p}(\tau) d \tau \leq\left(\frac{s}{r}\right)^{\alpha} u_{r}(s)+\varepsilon \frac{r}{\alpha+1}, \quad 0 \leq s<r<\infty .
$$

By further integrating in $r$, it follows that

$$
-1 \leq u(r)-u(s) \leq \frac{r^{1-\alpha}-s^{1-\alpha}}{1-\alpha} s^{\alpha} u_{r}(s)+\varepsilon \frac{r^{2}-s^{2}}{2(\alpha+1)}, \quad 0 \leq s<r<\infty,
$$

hence

$$
\frac{r^{1-\alpha}-s^{1-\alpha}}{1-\alpha} s^{\alpha}\left|u_{r}(s)\right| \leq 1+\varepsilon \frac{r^{2}-s^{2}}{2(\alpha+1)}, \quad 0 \leq s<r<\infty .
$$

Assume $\varepsilon \in(0,1)$. For $s \in[0,1]$, taking $r=2^{1 /(1-\alpha)}$ in $(2.9)$, we deduce that

$$
s^{\alpha}\left|u_{r}(s)\right| \leq c_{\alpha}:=1+\frac{2^{2 /(1-\alpha)}}{2(\alpha+1)}, \quad 0 \leq s \leq 1 .
$$

Setting $s_{\alpha}=\left(\frac{1-\alpha}{2 c_{\alpha}}\right)^{1 /(1-\alpha)} \in(0,1)$, it follows that

$$
u(s) \geq u(0)-\int_{0}^{s} c_{\alpha} \tau^{-\alpha} d \tau=1-\frac{c_{\alpha}}{1-\alpha} s^{1-\alpha} \geq 1 / 2, \quad 0<s \leq s_{\alpha} .
$$

But then applying (2.8) with $s=0$, we deduce that

$$
r^{\alpha} u_{r}(r) \leq \varepsilon \frac{r^{\alpha+1}}{\alpha+1}-\int_{0}^{r} \tau^{\alpha} u^{p}(\tau) d \tau \leq \varepsilon \frac{r^{\alpha+1}}{\alpha+1}-k_{\alpha, p}, \quad s_{\alpha}<r<\infty,
$$

where $k_{\alpha, p}=2^{-p} \int_{0}^{s_{\alpha}} \tau^{\alpha} d \tau$, hence

$$
u_{r}(r) \leq \varepsilon \frac{r}{\alpha+1}-k_{\alpha, p} r^{-\alpha}, \quad s_{\alpha}<r<\infty
$$

Consequently, we get

$$
\begin{aligned}
u(r) & \leq u\left(s_{\alpha}\right)+\varepsilon \frac{r^{2}-s_{\alpha}^{2}}{2(\alpha+1)}-k_{\alpha, p} \frac{r^{1-\alpha}-s_{\alpha}^{1-\alpha}}{1-\alpha} \\
& \leq 1-k_{\alpha, p} \frac{r^{1-\alpha}-s_{\alpha}^{1-\alpha}}{1-\alpha}+\varepsilon \frac{r^{2}}{2(\alpha+1)}, \quad s_{\alpha}<r<\infty .
\end{aligned}
$$

Taking $r=r(\alpha, p)>0$ large enough and then $\varepsilon=\varepsilon(\alpha, p)>0$ small enough, we get $u(r)<0$ : a contradiction. The conclusion follows.

\section{REFERENCES}

[1] C. Collot, F. Merle, P. Raphaël, On strongly anisotropic type II blow up, J. Amer. Math. Soc. (2020), https://doi.org/10.1090/jams/941.

[2] M. Del Pino, M. Musso, J. Wei, Geometry driven Type II higher dimensional blow-up for the critical heat equation (2017), Preprint arXiv: 1710.11461.

[3] M. Del Pino, M. Musso, J. Wei, Type II blow-up in the 5-dimensional energy critical heat equation, Acta Math. Sin. (Engl. Ser.) 35 (2019), 1027-1042.

[4] B. Gidas, Symmetry properties and isolated singularities of positive solutions of nonlinear elliptic equations, Nonlinear partial differential equations in engineering and applied science, Lecture Notes in Pure and Appl. Math. 54, Dekker, New York, 1980, pp. 255-273.

[5] Y. Giga, R. V. Kohn, Characterizing blowup using similarity variables, Indiana Univ. Math. J., 36 (1987), 1-40. 
[6] M. A. Herrero, J. J. L. Velázquez, Explosion de solutions des équations paraboliques semilinéaires supercritiques, C. R. Acad. Sc. Paris, 319, Série I (1994), 141-145.

[7] H. Matano, F. Merle, On nonexistence of type II blowup for a supercritical nonlinear heat equation, Comm. Pure Appl. Math. 57 (2004), 1494-1541.

[8] E. Mitidieri, S.I. Pohozaev, A priori estimates and blow-up of solutions of nonlinear partial differential equations and inequalities, Proc. Steklov Inst. Math. 234 (2001), 1-362.

[9] N. Mizoguchi, Type-II blowup for a semilinear heat equation, Adv. Differential Equations 9 (2004), 1279-1316.

[10] P. Quittner, Ph. Souplet, Superlinear parabolic problems. Blow-up, global existence and steady states. Second Edition. Birkhäuser Advanced Texts, 2019, 725 p.+xxii. ISBN: 978-3030-18220-5.

[11] R. Schweyer, Type II blow-up for the four dimensional energy critical semi linear heat equation, J. Funct. Anal. 263 (2012), 3922-3983.

[12] Ph. Souplet, S. Tayachi, Blow-up rates for nonlinear heat equations with gradient terms and for parabolic inequalities, Colloquium Mathematicum, 88 (2001), 135-154.

[13] F. B. Weissler, An $L^{\infty}$ blow-up estimate for a nonlinear heat equation, Comm. Pure Appl. Math., 38 (1985), 291-295. 